\title{
Fatores Socioeconômicos Associados à Obesidade Infantil em Escolares do Município de Carapicuíba (SP, Brasil)
}

\author{
Socioeconomic factors associated with obesity in schoolchildren from \\ Carapicuíba, SP, Brazil
}

\author{
EDNALVA MARIADE SOUSAESKENAZI ${ }^{1}$ \\ YARA CARDOSO COLETTO ${ }^{2}$ \\ LUCIMARATAMASO PAVANI AGOSTINI ${ }^{3}$ \\ FERNANDO LUIZAFONSO FONSECA ${ }^{4}$ \\ PAULAMIDORI CASTELO 4
}

\section{RESUMO}

Objetivo: o objetivo deste estudo foi avaliar a relação entre fatores socioeconômicos e o excesso de peso em escolares da rede pública do município de Carapicuíba (SP, Brasil). Material e Métodos: Foram incluídas 355 crianças (176 de cinco anos e 179 de 12 anos), selecionadas por amostragem aleatória simples, para a coleta das variáveis em estudo: sexo, escolaridade dos pais, renda familiar bruta, número de cômodos da casa e número de pessoas que habitavam o domicílio. O exame físico envolveu as medidas de peso, estatura e cálculo do índice de massa corporal (IMC) para a classificação das crianças em magreza severa, magreza, eutrofia, sobrepeso e obesidade (OMS, 2007). Os dados foram analisados por estatística descritiva, testes Quiquadrado, Kruskal-Wallis e correlação de Spearman. Resultados: Foram observados índices de excesso de peso e obesidade de $26,7 \%$ e $10,8 \%$ aos cinco anos, e $21,8 \%$ e $8,9 \%$ aos 12 anos, respectivamente. Os índices observados no grupo de cinco anos foram inferiores, enquanto os observados no grupo de 12 anos foram superiores aos índices nacionais. No grupo de cinco anos, o número de cômodos dos lares de crianças com obesidade foi significativamente maior que o de crianças que apresentavam eutrofia $(p=0,016)$; já no grupo de 12 anos, o IMC correlacionou-se positivamente com a escolaridade materna $(r=0,163 ; p=0,040)$. Conclusão: Os fatores socioeconômicos relacionaram-se com a presença de excesso de peso em escolares do município de Carapicuíba (SP, Brasil), devendo esta condição ser avaliada e abordada por meio de políticas e estratégias públicas de saúde em suas múltiplas dimensões.

\section{DESCRITORES}

Obesidade Infantil. Fatores Socioeconômicos. Criança.

\begin{abstract}
Objective: To evaluate the relationship between socioeconomic factors and overweight in children attending public schools in Carapicuíba, SP, Brazil. Material and Methods:The sample comprised 355 children (176 subjects 5years old, and 179 subjects 12years old), and the following variables were evaluated: sex, parental education, family income, number of rooms inthe household, and the number of people living in the household. Physical examination involved measurements of weight, height, and determination of the body mass index (BMI) to classify children into groups: severe underweight, underweight, normalweight, overweight and obesity. Data collected were analyzed using descriptive statistics, chi-square test, Kruskal-Wallis and Spearman correlation tests. Results:A prevalence of overweight and obesity of $26.7 \%$ and $10.8 \%$ in 5 -year-old children, and $21.8 \%$ and $8.9 \%$ in 12 -year-old children, respectively, was observed. The prevalence rates observed in 5-year-old children were lower than those of national surveys, whilethe rates observed among 12-year oldswerehigher. In the group of 5-year olds, the number of householdrooms of children with obesity was significantly higher than that of normalweight $(p=0.016)$ subjects. In the group of 12-year olds, BMI was positively correlated with maternal education $(r=0.163$, $p=0.040$ ). Conclusion: It is therefore concluded that socioeconomic factors were related with overweight and obesity in schoolchildren from Carapicuíba, SP, Brazil. This condition should be evaluated and addressed through public health policies and strategies in its multiple dimensions.
\end{abstract}

\section{DECRIPTORS}

Childhood Obesity.Socioeconomic Factors.Child.

\footnotetext{
Cirurgiã Dentista, Coordenadora de Saúde Bucal do Município de Carapicuíba. Carapicuíba. São Paulo. Brasil.

2 Aluna do curso de Farmácia e Bioquímica, Universidade Federal de São Paulo - UNIFESP, Diadema. São Paulo. Brasil.

3 Cirurgiã Dentista do Município de Carapicuíba. Carapicuíba. São Paulo. Brasil.
}

4 Professor Adjunto IV, Universidade Federal de São Paulo - UNIFESP. Diadema. São Paulo. Brasil. 
A obesidade tem sido descrita como um importante problema de saúde pública e ganhou bastante destaque no cenário epidemiológico mundial nos últimos anos. Sua prevalência aumentou nas últimas décadas em todo o mundo, inclusive em países emergentes como o Brasil, nos quais antes predominavam problemas relacionados à desnutrição ${ }^{1}$. Hoje, o Brasil vive um fenômeno chamado "transição nutricional", caracterizado pelo aumento das prevalências de sobrepeso e obesidade e diminuição da incidência de desnutrição. Esse fenômeno decorre da modernização e da crescente urbanização, frutos das mudanças econômicas, sociais e demográficas ocorridas na última metade do século $X X^{3}$.

De acordo com a OMS $(2010)^{2}$, estima-se que existam 43 milhões de crianças com menos de cinco anos com sobrepeso em todo o mundo. No Brasil,a Pesquisa de Orçamentos Familiares (POF 2008-2009) encontrou excesso de peso em $33,5 \%$ das crianças (uma a cada três) e obesidade em $14,3 \%$ das crianças de cinco a nove anos. Entre os indivíduos de 10 e 19 anos a pesquisa identificou que $20,5 \%$ apresentavam excesso de peso e $4,9 \%$, obesidade ${ }^{4}$.

O aumento da prevalência de sobrepeso e obesidade em idades cada vez mais precoces tem despertado a preocupação de pesquisadores e profissionais da área de saúde pelos danos e agravos à saúde provocados pelo excesso de peso ${ }^{1}$ e pelo fato de que o desenvolvimento da adiposidade celular nesse período é determinante para os padrões de composição corporal do indivíduo adulto $^{5}$. Sendo assim, o controle da obesidade infantil torna-se essencial, uma vez que sua prevalência pode resultar no desenvolvimento precoce de doenças crônicas que persistirão até a idade adulta, diminuição da qualidade de vida e custo elevado em cuidados de saúde ${ }^{6-8}$.

Preocupa também que as complicações da obesidade estão ocorrendo precocemente. Cerca de $60 \%$ das crianças entre cinco e 10 anos de idade têm pelo menos um fator de risco para doença cardiovascular (hipertensão arterial, dislipidemia, hiperinsulinemia, alteração do metabolismo da glicose, fatores pró-trombóticos) e $20 \%$ delas apresentam dois ou mais destes fatores ${ }^{9}$. O efeito do excesso de peso na adolescência sobre a morbidade e a mortalidade de adultos pode ser reflexo da deposição central de gordura, que afeta uma variedade de fatores de risco cardiovasculares, incluindo alterações na pressão arterial e perfis de lipoproteínas, as quais se relacionam com a formação de placas de ateroma ${ }^{8}$.
A etiologia desta condição, que pode trazer tantos agravos à saúde, é multifatorial e pode ser decorrente de uma combinação de fatores genéticos e ambientais e da condição socioeconômica, dentre elas, a renda e a educação. O nível socioeconômico interfere na disponibilidade de alimentos e no acesso à informação, bem como pode estar associado a determinados padrões de atividade física, constituindo-se, portanto, em fator importante que se associa à ocorrência de obesidade na infância ${ }^{10}$. Nos países emergentes, onde a disponibilidade de alimentos é um problema social, a obesidade na infância é mais prevalente nas classes socioeconômicas mais abastadas. Já nos países industrializados, nos quais até mesmo os indivíduos de baixa renda têm acesso ao alimento, pode-se encontrar uma menor prevalência de obesidade infantil nas classes de renda elevada, em decorrência do maior nível educacional que proporciona acesso à informação acerca de padrões dietéticos e de atividade física mais saudáveis ${ }^{10}$.

A escolaridade dos pais, especialmente a da mãe, interfere no desenvolvimento da obesidade nas crianças de várias formas, mas supõe-se que mais anos de estudo reflitam em maior renda e aquisição de alimentos de melhor qualidade. Por outro lado, a educação também poderia representar maior capacidade de incorporar recomendações de saúde e, assim, fazer opção por alimentos mais saudáveis, como é o caso da inclusão de frutas, verduras e legumes na dieta ${ }^{11}$.

Neste contexto, o presente estudo teve como objetivo verificar a relação entre fatores socioeconômicos e a prevalência de sobrepeso e obesidade em crianças de cinco e 12 anos, escolares da rede pública do município de Carapicuíba (SP, Brasil); município este inserido na Região Metropolitana de São Paulo e que possui 387.622 habitantes $^{12}$.

\section{MATERIAL E MÉTODOS}

\section{Amostra}

Trata-se de um estudo de corte transversal realizado a partir de um levantamento epidemiológico realizado no município de Carapicuíba (Estado de São Paulo, Brasil),entre os anos de 2012 e 2013, para avaliação da saúde bucal de escolares da rede pública do município com cinco e 12 anos de idade completos. As idades de cinco e 12 anos são de referência para a avaliação das condições de saúde bucal na infância ${ }^{13}$. A partir deste 
levantamento, foram coletados dados de interesse para o presente estudo.

As crianças foram selecionadas por amostragem aleatória simples a partir de uma listagem de todos os escolares com cinco e 12 anos de idade da rede pública municipal, os quais foram escolhidos de três em três da lista (para cinco anos) e de quatro em quatro (para os de 12 anos), para participar do estudo. Os pais ou responsáveis foram contatados e convidados a participar, sendo enviados, pelas crianças, os Termos de Consentimento Livre e Esclarecido (TCLE) e de Assentimento, além de um questionário endereçado aos pais para levantamento de dados demográficos. Foram incluídos na amostra final somente aquelas que retornaram o TCLE e termo de assentimento devidamente assinados e o questionário endereçado aos pais preenchido, totalizando 355 crianças, sendo 176 crianças de cinco anos e 179 crianças de 12 anos.

Este trabalho foi aprovado pelo Comitê de Ética em Pesquisa da Universidade Federal de São Paulo (UNIFESP - CAAE: 16984113.9.0000.5505).

\section{Anamnese e exame clínico}

A anamnese foi realizada por meio de inquérito (questionário) estruturado e enviado aos pais e/ou responsáveis legais. Por meio dele foram investigados: dados pessoais, histórico pré-natal, natal e pós-natal, escolaridade do pai e da mãe, número total de cômodos da residência da criança, número total de pessoas que habitavam o domicílio e renda familiar mensal (em reais).

A escolaridade do pai e da mãe foi mensurada em escala ordinal:analfabeto (0); ensino fundamental incompleto (1); ensino fundamental completo (2); ensino médio incompleto (3); ensino médio completo (4); ensino superior incompleto (5) e ensino superior completo (6).

O exame clínico foi realizado por duas pesquisadoras (EMSE e LTPA), no próprio ambiente escolar, em uma sala de aula reservada. Aavaliação antropométrica envolveu as medidas de peso e estatura com a utilização de balança digital e estadiômetro (Sanny, American Medical do Brasil Ltda., São Paulo, Brasil).

O índice de massa corporal $\left(\mathrm{IMC}=\mathrm{Kg} / \mathrm{m}^{2}\right)$ foi determinado a fim de dividir a amostra selecionada em cinco grupos de acordo com as curvas de referência IMCparaidade e sexo (5-19 anos) da OMS (2007), classificando as crianças em: magreza severa, magreza, eutrofia, sobrepeso e obesidade.

\section{Análise Estatística}

Os dados coletados foram analisados estatisticamente utilizando-se o pacote estatístico BioEstat 5.3 (Mamirauá, Belém, PA, Brasil). O teste de normalidade Kolmogorov-Smirnov foi utilizado para se verificar a distribuição das variáveis. Para aquelas que apresentaram desvio da distribuição normal, foram utilizados testes não-paramétricos. Adotou-se $p$-valor $<0,05$.

A estatística descritiva consistiu de médias, desvio-padrão, medianas e porcentagens. A proporção de meninos e meninas entre os grupos foi verificada por meio do teste Qui-quadradopartição. As diferenças entre os grupos para variáveis contínuas foram verificadas utilizando-se o teste Kruskal-Wallis (e pós-teste de Dunn). O teste de correlação de Spearman foi utilizado para se verificar a correlação entre o IMC e a escolaridade materna e paterna.

\section{RESULTADOS}

A proporção de crianças do sexo feminino e masculino entre os grupos classificados como tendo magreza severa, magreza, eutrofia, sobrepeso e obesidade não diferiu significativamente em ambas as idades, mostrando homogeneidade na composição dos grupos.

Os dados coletados das crianças de cinco anos estão apresentados na tabela 1 quanto à escolaridade materna e paterna, sendo ilustrada a porcentagem de pais com ensino fundamental completo (acima de oito anos de estudo).A comparação entre os grupos mostrou uma diferença estatisticamente significativa no número de cômodos do domicílio entre crianças com eutrofia e com obesidade ( $p=0,016)$. Já a renda familiar e o número de pessoas que habitavam o domicílio não diferiram significativamente entre os grupos.

Os dados demográficos das crianças de 12 anos estão apresentados na tabela 2. Não foi observada diferença estatisticamente significativa nas variáveis renda familiar, número de cômodos e número de moradores do domicílio entre os grupos de crianças com 12 anos.

A tabela 3 mostra os valores dos coeficientes de correlação obtidos entre as escolaridades materna e paterna e o IMC, utilizando-se o teste de correlação de Spearman. Nesta análise, observouse uma correlação positiva significativa entre escolaridade materna e IMC aos 12 anos ( $r=0,163$; $p=0,040)$, ou seja, quanto maior a escolaridade materna, maior o IMC da criança. 


\begin{tabular}{|c|c|c|c|c|c|c|c|c|c|}
\hline & $\begin{array}{c}n \\
(\% \\
\text { total) }\end{array}$ & Gênero & $\begin{array}{c}\text { Idade } \\
\text { (meses) }\end{array}$ & $\begin{array}{c}\text { IMC } \\
\left(\mathrm{Kg} / \mathrm{m}^{2}\right)\end{array}$ & $\begin{array}{c}\text { Escolaridade } \\
\text { materna } \\
\text { (acima de } \\
\text { oito anos) }\end{array}$ & $\begin{array}{c}\text { Escolaridade } \\
\text { paterna } \\
\text { (acima de } \\
\text { oito anos) }\end{array}$ & $\begin{array}{l}\text { Renda } \\
\text { familiar } \\
\text { (Reais) }\end{array}$ & $\begin{array}{c}\text { Número } \\
\text { de } \\
\text { cômodos } \\
\text { no } \\
\text { domicílio }\end{array}$ & $\begin{array}{c}\text { Número } \\
\text { de } \\
\text { moradores } \\
\text { no } \\
\text { domicílio }\end{array}$ \\
\hline & & & $\begin{array}{l}\text { Média } \\
\text { (DP) }\end{array}$ & $\begin{array}{l}\text { Média } \\
\text { (DP) }\end{array}$ & $(\%)$ & $(\%)$ & $\begin{array}{c}\text { Mediana } \\
(25- \\
75 \%)\end{array}$ & $\begin{array}{l}\text { Mediana } \\
(25-75 \%)\end{array}$ & $\begin{array}{l}\text { Mediana } \\
(25-75 \%)\end{array}$ \\
\hline $\begin{array}{l}\text { Magreza } \\
\text { severa }\end{array}$ & $\begin{array}{c}5 \\
(2,8)\end{array}$ & 3 웅 & $\begin{array}{l}69,6 \\
(1,5)\end{array}$ & $\begin{array}{l}10,5 \\
(1,2)\end{array}$ & 20,0 & 50,0 & $\begin{array}{l}1020,0 \\
(810,0- \\
1425,0)\end{array}$ & $\begin{array}{c}4,0 \\
(3,0-5,0)\end{array}$ & $\begin{array}{c}6,0 \\
(4,5-7,0)\end{array}$ \\
\hline Magreza & $\begin{array}{c}5 \\
(2,8)\end{array}$ & $3+20^{\pi}$ & $\begin{array}{l}69,2 \\
(2,4)\end{array}$ & $\begin{array}{l}12,5 \\
(0,3)\end{array}$ & 50,0 & 50,0 & $\begin{array}{c}720,0 \\
(550,0- \\
1000,0)\end{array}$ & $\begin{array}{c}4,0 \\
(4,0-4,0)\end{array}$ & $\begin{array}{c}5,0 \\
(4,3-5,8)\end{array}$ \\
\hline Eutrofia & $\begin{array}{c}119 \\
(67,6)\end{array}$ & $\begin{array}{l}51 \text { \% } \\
680^{\circ}\end{array}$ & $\begin{array}{l}69,3 \\
(2,2)\end{array}$ & $\begin{array}{l}15,3 \\
(0,9)\end{array}$ & 70,3 & 60,0 & $\begin{array}{c}800,0 \\
(620,0- \\
1134,5)\end{array}$ & $\begin{array}{c}3,0^{*} \\
(2,0-4,0)\end{array}$ & $\begin{array}{c}5,0 \\
(4,0-5,0)\end{array}$ \\
\hline Sobrepeso & $\begin{array}{c}28 \\
(15,9)\end{array}$ & $\begin{array}{l}12 \text { q } \\
16{ }^{\lambda}\end{array}$ & $\begin{array}{l}69,4 \\
(1,6)\end{array}$ & $\begin{array}{l}17,7 \\
(0,6)\end{array}$ & 87,5 & 70,8 & $\begin{array}{c}825,0 \\
(620,0- \\
1200,0)\end{array}$ & $\begin{array}{c}4,0 \\
(3,0-4,0)\end{array}$ & $\begin{array}{c}5,0 \\
(3,5-6,5)\end{array}$ \\
\hline Obeso & $\begin{array}{c}19 \\
(10,8)\end{array}$ & $10+9{ }^{\pi}$ & $\begin{array}{l}69,2 \\
(1,3)\end{array}$ & $\begin{array}{l}21,4 \\
(2,4)\end{array}$ & 55,6 & 60,0 & $\begin{array}{c}900,0 \\
(635,0- \\
1200,0)\end{array}$ & $\begin{array}{c}4,0^{*} \\
(3,0-5,0)\end{array}$ & $\begin{array}{c}4,0 \\
(3,0-6,0)\end{array}$ \\
\hline
\end{tabular}

IMC, índice de massa corporal; DP, desvio padrão.

${ }^{*} p<0,05$ (teste Kruskal-Wallis e Dunn pós-teste).

Os dados demográficos das crianças de 12 anos estão apresentados na tabela 2. Não foi observada diferença estatisticamente significativa nas variáveis renda familiar, número de cômodos e número de moradores do domicílio entre os grupos de crianças com 12 anos.

\begin{tabular}{|l|c|c|c|c|c|c|c|c|c|}
\hline \multicolumn{1}{|c|}{ Tabela 2. Dados demográficos das crianças com 12 anos (n=179), segundo categorias de IMC. } \\
Carapicuíba, SP, 2014.
\end{tabular}

p>0,05 (teste Kruskal-Wallis e Dunn pós-teste). 


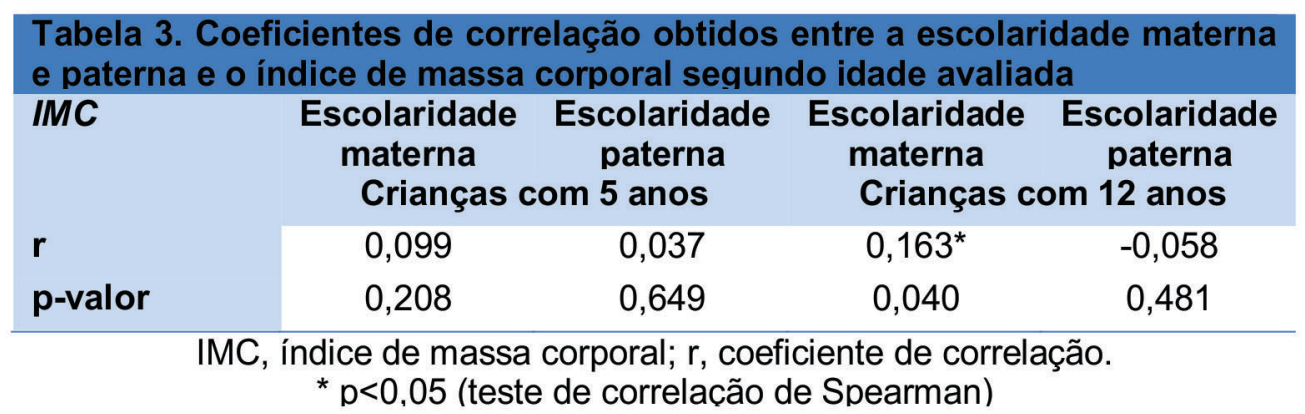

\section{DISCUSSÃO}

O município de Carapicuíba possui área de $34,55 \mathrm{~km}^{2}$ e está inserido na Região Metropolitana do Estado de São Paulo, com densidade demográfica de $10.698,32$ habitantes $/ \mathrm{km}^{214}$. De acordo com a Fundação Sistema Estadual de Análise de Dados de SP, o município possui 387.622 habitantes e a taxa de analfabetismo do município é de $4,36 \%$, semelhante à do Estado de SP $(4,33 \%)$ e superior à taxa em sua Região Governamental $(3,60 \%)^{12}$. O Produto Interno Bruto (PIB) per capita do município é de $R \$ 11.079,32$ (onze mil e setenta e nove reais e trinta e dois centavos $)^{14}$, ficando na posição $606^{\mathrm{a}}$ dos 645 municípios do Estado de São Paulo.

Das crianças com cinco anos incluídas no estudo, $26,7 \%$ apresentou excesso de peso, índice este abaixo do encontrado naPesquisa de Orçamentos Familiares(POF 2008-2009), onde o excesso de peso foi observado em $33,5 \%$ das crianças brasileiras da zona urbana entre cinco e nove anos. Já em relação à obesidade, $10,8 \%$ das crianças incluídas no estudo apresentaram obesidade, índice também abaixo do nacional, que foi de $14,3 \%$.

Quanto aos indivíduos de 12 anos incluídos no estudo, $21,8 \%$ apresentavam excesso de peso, índice acima do nacional(POF 2008-2009) em que o excesso de peso foi observado em $20,5 \%$ dos indivíduos brasileiros da zona urbana entre 10 e 19 anos. Entre as crianças com 12 anos, 8,9\% delas apresentavam obesidade, quase o dobro do índice nacional (POF 2008-2009), o que pode sugerir uma tendência de ganho de peso durante os anos que antecedem a puberdade neste município, embora tal inferência seja feita com certa cautela, uma vez que este estudo tem característica de corte transversal.

De acordo com dados de 2013 colhidos do Sistema de Vigilância Alimentar e Nutricional ${ }^{15}$, dos
856 adolescentes do município de Carapicuíba acompanhados pelo SUS, $23,33 \%$ apresentavam sobrepeso, $8,37 \%$ apresentavam obesidade e $1,67 \%$ apresentavam obesidade severa. Portanto, $33,37 \%$ dos adolescentes acompanhados apresentavam excesso de peso para a idade. Notase que a prevalência de obesidade observada entre os sujeitos de 12 anos $(8,9 \%)$ foi bem próxima ao índice encontrado no SISVAN (2013) para os adolescentes do município de Carapicuíba. Para crianças entre cinco e 10 anos de idade não há dados disponíveis no sistema mencionado. Notase que, quando comparados aos índices obtidos no território nacional ${ }^{15}$ e na Região Sudeste, o Estado de São Paulo e o Município de Carapicuíba apresentam porcentagens superiores de sobrepeso entre os adolescentes acompanhados pelo SUS.

Emestudo transversal realizado com adolescentes de 10 a 18 anos, matriculados em escolas públicas estaduais e municipais do município de Jundiaí (SP) no ano de 2005, observouse que a prevalência de excesso de peso foi de $25 \%{ }^{16}$, índice superior ao encontrado no município de Carapicuíba, quase 10 anos antes. Este fato pode ser explicado, em parte, devido à diferença entre as condições econômicas dos dois municípios. De acordo com dados do IBGE (2011), o PIB per capita do município de Jundiaí $(\mathrm{R} \$ 58.351,18)$ é quase seis vezes maior do que o de Carapicuíba ( $R \$ 11.079,32)$. Outro estudo conduzido no município de Santos (SP $)^{17}$, envolveu 10.822 crianças de 7 a 10 anos de idade de escolas públicas e particulares e mostrou uma prevalência de obesidade de $18 \%$, índice acima dos encontrados neste estudo. De acordo com dados do IBGE (2011), o PIB per capita do município de Santos $(R \$ 75.194,67)$ é quase sete vezes maior que o de Carapicuíba ( $R$ \$ $11.079,32)$.

O aumento acelerado dos índices nacionais de sobrepeso e obesidade na população infantil parece estar relacionado ao crescimento do poder 
aquisitivo das famílias de menor renda, aumento da escolaridade materna e melhorias na cobertura de serviços básicos de saúde e saneamento ${ }^{18}$. Corroborando estas evidências, o presente estudo observou que aos cinco anos as crianças obesas moravam em domicílios com maior número de cômodos. Ainda, entre crianças de 12 anos, o IMC correlacionou-se positivamente com a escolaridade materna. De acordo com o índice nacional ${ }^{4}$, o aumento na renda familiar relaciona-se com o excesso de peso na população brasileira urbana; o maior poder aquisitivo permite $\mathrm{o}$ acesso a alimentos industrializados com alto teor de energia, que associado à diminuição na prática de exercícios físicos tem contribuído para o aumento nos índices de obesidade ${ }^{19}$. Com o aumento do grau de instrução e consequente participação das mulheres no mercado de trabalho, estas dispõem de menos tempo para o cuidado familiar, facilitando a influência publicitária e de terceiros na aquisição de hábitos alimentares errôneos ${ }^{20}$. Além disto, é provável que o aumento na escolaridade materna nas famílias brasileiras de menor renda tenha sido recente, não havendo tempo suficiente para o contato e incorporação de hábitos mais saudáveis.

Corroborando estes achados, foi encontrado no município de Piracicaba (SP) um índice de excesso de peso de $39,2 \%$ entre os 309 escolares da rede pública com oito, nove e 10 anos de idade incluídos no estudo ${ }^{21}$, verificou-se também que aquelas crianças com nível socioeconômico mais elevado tinham mais chances de apresentar sobrepeso/obesidade. Na Pesquisa Nacional de Saúde do Escolar (2009)22, foram avaliados 60.973 adolescentes do $9^{\circ}$ ano do ensino fundamental de escolas públicas e privadas de todas as capitais brasileiras, entre março e junho de 2009. Os resultados obtidos demonstraram que as prevalências de déficits em altura e peso foram maiores entre os jovens cujas mães apresentavam menos tempo de estudo, enquanto as prevalências de excesso de peso foram superiores naqueles cujas mães tinham mais de 10 anos de estudo. Outros estudos conduzidos em municípios brasileiros também encontraram relação positiva entre escolaridade materna e excesso de peso ${ }^{20,23}$, demonstrando que, ainda hoje, o maior nível educacional da mãe não garante maior conhecimento a respeito de hábitos alimentares corretos.

Já as demais variáveis avaliadas, como escolaridade paterna, número de pessoas que habitam o domicílio e renda familiar, não diferiram entre os grupos em ambas as idades. As diversas formas de abordagem das condições socioeconômicas familiares se fazem necessárias para aumentar a sensibilidade do questionário realizado, contornando vieses de informação, como a omissão de dados e a informação de dados incompletos ou incorretos ${ }^{24}$. A renda mensal familiar, por exemplo, tem caráter variável e flutuante; como foi autodeclarada, há uma tendência a enfatizar ou exagerar uma medida desejável pelo entrevistado, gerando uma possibilidade de viés neste tipo de mensuração ${ }^{25}$.

Este panorama é bastante diferente nos países industrializados, onde estudos têm mostrado uma maior prevalência de obesidade nos segmentos populacionais de pior nível socioeconômico ${ }^{23}$, onde existe fácil acesso a alimentos calóricos mas pobres em termos nutricionais. Na Alemanha, um estudo mostrouque crianças com seis anos, filhos de mães sem escolaridade, apresentavam riscos três vezes maiores de apresentarem obesidade, quando comparados com filhos de mães com 13 anos de estudo ${ }^{11}$. Nos Estados Unidos (EUA), medidas de saúde pública têmsidoimplementadas, como o aumento de impostos para os produtos de bebidas açucaradas, anúncios nutricionais e a limitação do mercado a crianças ${ }^{26}$. Ainda, uma série de medidas legais vêm sendo elaboradas e aprovadas ao longo dos anos, como a proibição da venda de fast-foods acompanhada de brinquedos, venda de bebidas altamente calóricas, como refrigerantes, em volumes superiores a 473 mililitros ${ }^{27}$, além da restriçãoda venda de alimentos antes e apóso café da manhã e no intervalo do almoço em todo o recinto escolar. Destacam-se, ainda, a implementação de medidas educativas que 
têm como finalidade apoiar autoridades locais no desenvolvimento de trabalhos relativos às políticas de nutrição e atividades físicas ${ }^{28}$. Um estudo recente mostrou que a obesidade infantildiminuiu significativamente nos EUA ${ }^{29}$, passando de $14 \%$ em 2003-2004 para de 8,3\% em 2011-2012 entre crianças de dois e cinco anos de idade.

No Brasil, algumas medidas legais foram aprovadas recentemente, visando à diminuição da obesidade na infância, dentre as quais podemos citar a Lei no 12.283, de 22 de fevereiro de 2006, que Institui a Política de Combate à Obesidade e ao Sobrepeso("São Paulo Mais Leve") e a Lei estadual $n^{\circ} 4508$, de 11 de janeiro de 2005, que proíbe a comercialização, aquisição, confecção e distribuição de produtos que colaborem para a obesidade infantil em bares, cantinas e similares instalados em escolas públicas e privadas do estado do Rio de Janeiro. Por outro lado, medidas de caráter educativo e informativo, inseridas no currículo escolar e nos meios de comunicação de massa, assim como o controle da propaganda de alimentos que não são saudáveis, dirigidos principalmente ao público infantil, são ações que ainda devem ser propostas e mais bem trabalhadasno território brasileiro ${ }^{30}$.

Dessa forma, os achados do presente estudo corroboram os resultados de estudos prévios que evidenciam a necessidade de políticas públicas de saúde associadas às medidas e estratégias educativas que propiciem a incorporação de hábitos saudáveis, como a prática de exercícios físicos e a inclusão de frutas, verduras e legumes na dieta familiar ${ }^{11}$.

\section{CONCLUSÃO}

Os achados do presente estudo nos permitem concluir que alguns fatores socioeconômicos pesquisados relacionaram-se com a presença de excesso de peso em escolares da rede pública do município de Carapicuíba (SP, Brasil). Entre as crianças de cinco anos, o número de cômodos foi significativamente maior nos domicílios de crianças que apresentavam obesidade,entre as crianças de 12 anos, observou-se que quanto maior a escolaridade materna, maior era o índice de massa corporal da criança.

Sendo assim, esta condição deve ser avaliada e abordada por meio de políticas e estratégias públicas de saúde em suas múltiplas dimensões, mas que envolvam medidas de caráter educativo e informativo.

\section{REFERÊNCIAS}

1. Enes CC, Slater B. Obesidade na adolescência e seus principais fatores determinantes. RevBrasEpidemiol. 2010; 13(1):163-71.

2. World Health Organization (WHO). Childhood overweight and obesity on the rise. 2010. Lang RMF, Nascimento AN, Taddei JAAC. A transição nutricional e a população infanto-juvenil: medidas de proteção contra o marketing de alimentos e bebidas prejudiciais à saúde. Nutrire. 2009; 34(7):217-29

3. Instituto Brasileiro De Geografia e Estatística. Pesquisa de orçamentos familiares (POF) 2008- 2009. Antropometria e estado nutricional de crianças, adolescentes e adultos no Brasil. Rio de Janeiro: IBGE; 2010.

4. Soares LD, PetroskiEL. Prevalência, fatores etiológicos e tratamento da obesidade infantil. Rev Bras Cineantropom DesempenhoHum. 2003; 5(1):6374

5. Poeta LS, Duarte MFS, Giuliano ICB. Qualidade de vida relacionada à saúde de crianças obesas. RevAssocMed Bras. 2010;56(2):168-72

6. Francischi RPP, Pereira LO, Freitas CS, Klopfer M, Santos RC, Vieira P, et al. Obesidade: atualização sobre etiologia, morbidade e tratamento. Rev Nutr. 2000 13(1):17-28.

7. Williams CL, Hayman LL, Daniels SR, Robinson TN, Steinberger J, Paridon S, Bazzarre T. Cardiovascular health in childhood: A statement for health professionals from the Committee on Atherosclerosis, Hypertension, and Obesity in the Young (AHOY) of the Council on Cardiovascular Disease in the Young, American Heart Association. Circulation. 2002; 106(1): 143-60.

8. Amemiya S,Dobashi K, Urakami T ,Sugihara S , Ohzeki T, Tajima N. A síndrome metabólica em jovens. Pediatr Diabetes. 2007; 8(9):48-54. 
9. Silva GAP, Balaban G, Motta MEFA. Prevalência de sobrepeso e obesidade em crianças e adolescentes de diferentes condições socioeconômicas. RevBras Saúde Mater Infant. 2005; 5(1):53-9.

10. Schuchl, DeCastro TG, De Vasconcelos FA, Dutra CL, GoldaniMZ. Excess weight in preschoolers: prevalence and associated factors. J Pediatr (Rio J). 2013;89(2):179"88.

11. Portal de Estatísticas do Estado de São Paulo [Internet]. Fundação Sistema Estadual de Análise de Dados [citado em 28/07/2015]

12. World Health Organization - WHO. Oral health surveys : basic methods. 4th ed. Geneva: World Health Organization, 1997.

13. Instituto Brasileiro de Geografia e Estatística [Internet] PIB per capita a preços correntes 2012.

14. Brasil - Ministério da Saúde [Internet]. SISVAN [citado em 28/07/2015]. Disponível em:http://200.214.130.94/ nutricao/sisvan.php

15. VanzelliA, Castro C, Pinto M, Passo S. Prevalência de sobrepeso e obesidade em escolares da rede pública do município de Jundiaí, São Paulo. Rev Paul Ped.2008; 26(1):48-53.

16. Costa RF, Cintra IP, FisbergM. Prevalência de sobrepeso e obesidade em Escolares da Cidade de Santos, SP.ArqBrasEndocrinolMetab. 2006; 50(1): 60-7.

17. Monteiro CA, Benicio MHD, Konno SC, Da Silva ACF Delima ALL, Conde WL. Causas do declínio da desnutrição infantil no Brasil, 1996-2007. Rev Saúde Pública 2009;43(1):35-43.

18. Halpern Z, Rodrigues MDB. Obesidade infantil. In: Nunes MA, Appolinário JC, Galvão AL, Coutinho W. Transtornos alimentares e obesidade. 2a ed. Porto Alegre: Artmed; 2006. p. 283-288.

19. Leal VS, Lira PIC, Oliveira JS, Menezes RCE, Sequeira LAS, Arruda Neto MA, et al.Excesso de peso em crianças e adolescentes no Estado de Pernambuco, Brasil: prevalência e determinantes. Cad Saúde Pública. 2012;28(6):1175-82.

20. Araujo DS, Marquezin M, Barbosa TS, Fonseca F, Fegadolli C, Castelo PM. Assessment of quality of life, anxiety, socio-economic factors and caries experience in Brazilian children with overweight and obesity. Int $\mathrm{J}$ Dent Hyg. 2016 Oct 4.
21. Instituto Brasileiro de Geografia e Estatística. Pesquisa Nacional de Saúde Escolar. Rio de Janeiro: IBGE; 2009.

22. Guimarães LV, Barros MBA, Martins MSAS, Duarte EC. Fatores associados ao sobrepeso em escolares. Rev Nutr. 2006; 19(1):5-17.

23. Richardson RJ. Pesquisa social: métodos e técnicas. 3. ed. São Paulo: Atlas, 334 p.

24. Loughnan S, Kuppens P, Allik J, Balazs K, DeLemus S, Dumont $\mathrm{K}$ et al. Economic inequality is linked to biased self-perception. Psychol Sci. 2011; 22(10):1254-8.

25. Polesel MG. A redução do consumo de bebidas adoçadas com açúcar previne obesidade e Diabetes. Centro de Diabetes Curitiba. Disponível em: http:// www.centrode diabetescuritiba.com.br/artigos/areducao-do-consumo-de-bebidas-adocadas-comacucar-previne-obesidade-e-diabetes/. Acesso em: 20 de julho de 2015

26. Associação Brasileira para o estudo da obesidade e da Síndrome Metabólica (ABESO). Refrigerantes Grandes São Proibidos em Nova York. Disponível em: http://www.abeso.org.br/lenoticia/925/ refrigerantes+grandes+sao+proibidos+em+nova+york.shtml. Acesso em: 09 out. 2014.

27. America's Move to Raise A Healthier Generation of Kids (Let's Move!).Disponívelem: http://www.letsmove.gov/ . Acessoem: 09 out. 2014.

28. Ogden CL, Carroll MD, Kit BK, FlegalKM. Prevalence of childhood and adult obesity in the United States, 20112012. JAMA 2014; 311(8):806-14.

29. Oliveira $C L$, Fisberg $M$. Obesidade da Infância e Adolescência - Uma Verdadeira Epidemia. Arq.Bras. EndocrinolMetab.2003; 47(2):107-108.

CORRESPONDÊNCIA

Paula Midori Castelo

R. São Nicolau, $n^{\circ} 210$ - 1. andar

CEP: 09913-030

Diadema - SP - Brasil

E-mail: pcastelo@yahoo.com 